\title{
Computer module for scheduling of transportation of composite beam bridge structures
}

\author{
Wojciech Bożejko ${ }^{1}$, Zdzisław Hejducki ${ }^{1{ }^{*},}$, Mariusz Uchroński ${ }^{1}$, Mieczysław Wodecki $^{2}$ \\ ${ }^{1}$ Wrocław University of Science and Technology, Wyb. Wyspiańskiego 27, 50-370 Wrocław, Poland \\ ${ }^{2}$ University of Wrocław, P1. Uniwersytecki 1, 50-137 Wrocław, Poland
}

\begin{abstract}
The paper presents the theoretical basis, an algorithm and a computer module system supporting scheduling of transportation and assembly of structures of the composite beam bridge implemented in a Just In Time system (JIT). Tabu search method has been used in the optimization procedure.
\end{abstract}

\section{Introduction}

During an organization of work in the JIT system it is necessary to synchronize the supply (prefabrication transport) with the installation, avoiding the need of storage. It requires solving of many difficult (strongly NP-hard) optimization problems at the same time. There are currently no optimal algorithms for solving the problems in polynomial time. Therefore, we propose to use a fast approximate algorithm of polynomial time computational complexity - tabu search method.

The paper concerns an issue of transportation of prefabricated bridge components (by rail and road) to ensure collision-free paths. In recent years, there have been developed new technological solutions, using polymer composites (Fibre Reinforced Polymers called for short FRP).They consist of the composites of plastics reinforced with various fibers. Most of them are carbon, glass or aramid fibers. Due to a number of the beneficial properties, such materials find more and more practical applications, including bridge construction.

The establishment of the EN 13706 European standard for the production of polymer composites with the use of pultrusion method enabled the development of new technology, methods of design and installation of bridge load-bearing components. The proposed technology provides:

- Lower costs of construction associated with prefabrication and low weight of elements,

- Lower costs of maintenance during the exploitation of the structures (elements surfaces do not corrode and do not require painting),

- Less disruption to traffic flow by reducing the installation time.

It is assumed that the assembly of composite structures takes place on concrete support with bearings in the Trans-IND developed technology and construction system. In this system, work division structure includes:

*Corresponding author: zdzislaw.hejducki@pwr.edu.p1 
1. Transportation of beams from the factory to the installation site.

2. Installation of beams.

3. Transport of mechanical fasteners and resin for fastening designed to connect elements at the site.

4. Transport of modular composite bridges to be installed at the site.

5. Leveling and connecting bridge decks.

6. Connecting of bridges and beams.

7. Execution of concrete slab on the surface of bridges.

8. Building of hard asphalt pavement.

9. Installation of the bridge equipment (barriers).

Figures 1-3 depict some phases of bridge construction using composite structures.

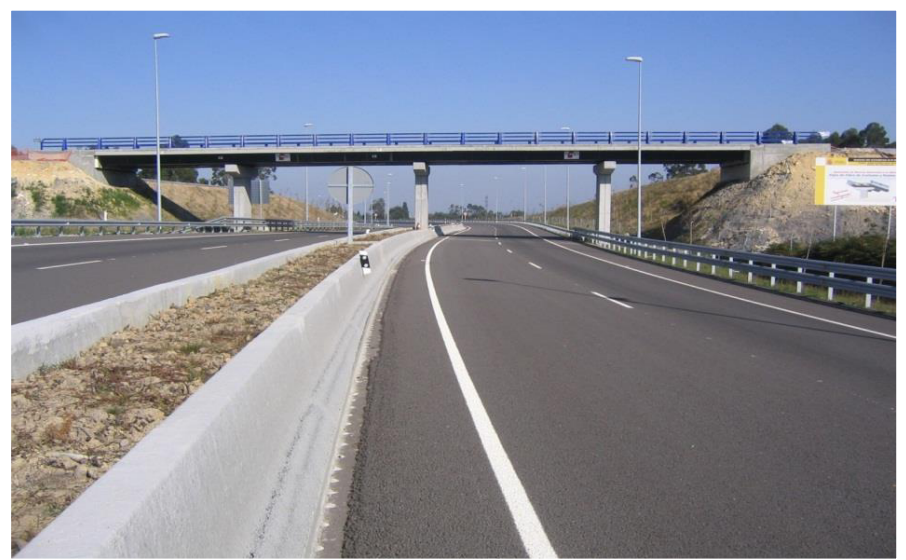

Fig. 1. Concept of a composite bridge (by BSiR Mostostal SA).

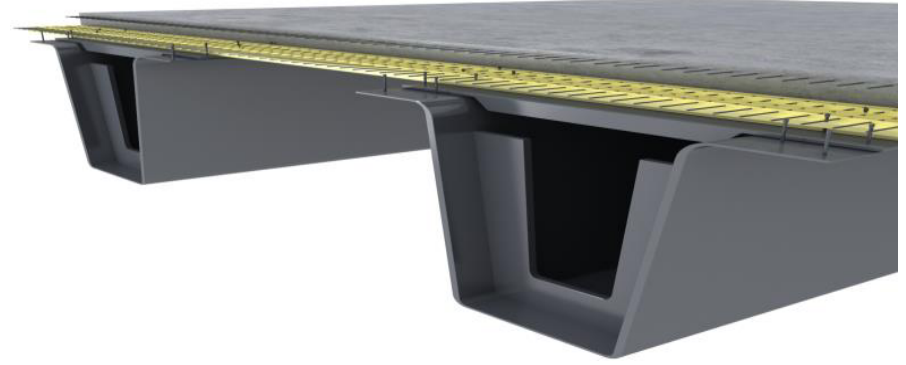

Fig. 2. Scheme of beams and slab (by BSiR Mostostal SA).

In the paper [1] there was, among other issues, the problem of scheduling of bridge structure construction described, including girders installation. This paper presents a description of the problem along with the organizational and technical considerations, the theoretical basis for model building, the construction algorithm based on the tabu search method, and the results of computational experiments. On the basis of the schedule set by the algorithm for girders installation there are time windows determined, i.e. the earliest and the latest required delivery of individual beams. At their base there is determined optimal (or nearly optimal) schedule of transportation from the place of prefabrication to the construction site. A model of this problem and the method of its solution are presented below. 


\section{Girder transportation problem}

One of the important aspects of the installation of bridge structure, using a load-bearing components made of composites, is the transportation and fitting of bridge girders. On the basis of developed and agreed with all subcontractors the schedule of construction: dates and order of delivery of beams (in the order of technology) is determined. The basic assumption of the developed methodology relies on a specific logistic condition, that is, assumption concerning assembly and transport in the Just In Time system. As a result, there is no storage capacity for girders on site - possibly only on vehicles. Therefore, the girders should be directly placed on the supports. The problem of optimal transportation of beams (in short the problem TB) is to determine such delivery dates of girders that they minimize the assumed criterion (e.g., the cost of non-compliance with delivery dates, the number of necessary vehicles, etc.). The following restrictions must be met:

1. Beams should be delivered in accordance with the order of construction (technology policy),

2. The vehicle can carry only a single beam (or part of beams) at once,

3. After loading, the beam can be removed immediately prior to installation only.

In the following part, we present some variants of beam transportation problem.

\subsection{Transportation with the earliest and the latest delivery dates}

We assume that on the basis of work implementation dates and technology requirements there has been a detailed schedule of construction and assembly works determined. Among many other issues, it contains the starting and ending dates of girders installation. On this basis, there are the earliest and the latest possible delivery dates of individual girders determined (taking into account also the technological order). In determining the latest delivery date the unloading time should be taken into consideration, so that launching of girder installation take place according to the schedule. It is recommended, moreover, to introduce a small buffer in time, taking into account the fact that in the course of proceedings some slight complications may occur. The earliest delivery date result from the fact that the beams cannot be stored on site. Therefore, the vehicle which arrives too early cannot be discharged before that date. The earliest and latest date of delivery form so called time window. This is the time interval in which the delivery generates no extra cost. A mathematical model of this problem is presented below.

Let:

$$
B=\left\{B_{1}, B_{2}, \ldots, B_{m}\right\}
$$

Be a set of girders provided for installation which have to be delivered from the prefabrication site to the construction site. For any beam $B_{i} \in B$ we introduce the following symbols:

$z_{i}-$ loading time,

$t_{i}-$ time of transportation to building site,

$r_{i}$ - time of unloading at construction site,

$p_{i}$ - returning time (time taken from the construction site to the prefabrication site),

$e_{i}-$ earliest required delivery to construction site,

$d_{i}$ - latest required delivery to construction site,

$v_{i}$ - penalty function coefficient of too early delivery to the construction site,

$w_{i}$ - penalty function coefficient of too late delivery to the construction site. 
On the basis of the schedule of construction and assembly works there was the order of girders installations determined (delivery to the construction site). To prove the statement, we assume that this is the sequence $\left(B_{1}, B_{2}, \ldots, B_{m}\right)$. We also assume that the loading of the first beam starts at zero moment. By $S_{i}$ we denote the delivery date of $B_{i}$ girder to the site. The dates $S_{1}, S_{2}, \ldots, S_{m}$ must satisfy constraints (a)-(c).

If the transport and assembly took place on a continuous basis, the date of delivery of the girder $B_{i}$ is equal to

$$
S_{i}=\sum_{i=1}^{n}\left(z_{j}+t_{j}+r_{j}+p_{j}\right)+z_{i}+t_{i}
$$

Girders transportation problem boils down to determining of delivery dates $S_{1}, S_{2}, \ldots, S_{m}$ satisfying constraints (a) - (c) that optimize certain adopted optimization criterion. In JIT system it is usually minimization of a number of exceeded delivery dates, the sum of penalties for late delivery or for maximum delays, etc.

\section{Support system for scheduling of transportation and assembly of composite elements}

Support system for scheduling transportation and installation of prefabricated composite bridge structure elements was written in $\mathrm{C}++$ Microsoft Windows. It consists of three main modules, of scheduling:

1. Construction works.

2. Transportation.

3. Works and transport in uncertain conditions.

After booting the system there is the main window displayed containing various system options: Scheduling, Transportation, Uncertainty and About. Figure 4 shows the window, after clicking the scheduling of construction works option (Scheduling).

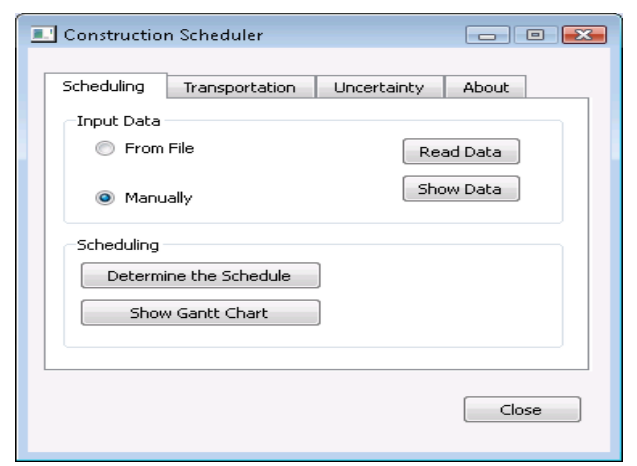

Fig. 3. Main window, scheduling of construction works.

\subsection{Transportation scheduling}

Transportation scheduling module allows us to determine the delivery dates of girders to the construction site. These terms are based on the basis of the construction schedule, set in the Scheduling module. For each girder there is a time window determined (the earliest and latest date) in which the girder must be delivered to the construction site. The theoretical basis of the transportation problem was presented in Chapter 2. There was an algorithm based on the tabu search method [2] used to solve this problem. Installation of girders was 
done in the JIT system, therefore, as optimization criterion there was minimization of the sum of the penalties for late delivery (1) adopted.

After selecting the Transportation option from the main window (Figure 4) there are shown the menu options for this module which allow us, among other things, to introduce and correct data (they were thoroughly described in the previous chapter). The parameters necessary for determining the schedule of girders delivery to the construction site can be entered manually or read from a text file. The window displaying transportation scheduling module was shown in Figure 4.

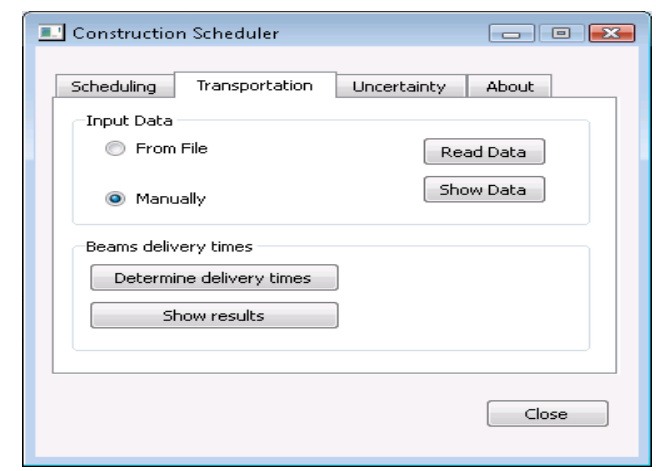

Fig. 4. Beam transportation scheduling window.

The individual modules, in particular the optimization algorithms were tested on the examples taken from the construction practice and from the literature. The experimental results were entirely satisfactory. The optimum solution, or little different from the optimal (average relative error for a large set of examples in the literature did not exceed 3\%) was obtained in a very short period of time (maximum in a few seconds).

\section{Conclusions}

Modules of the support system for scheduling of construction projects, presented in the paper, provides a simple-to-use graphical user interface. This allows for a rapid determination of solutions for problems of different sizes, parameters and characteristics. The obtained results are presented in graphic form (Gantt chart) or in the form of text (tables of starting and ending dates of individual work performance). Application of a fast approximate algorithm (tabu search) for scheduling of construction projects allows us to obtain high quality solutions (in terms of the value of the objective function) in a short time.

\section{References}

1. W. Bożejko, Z. Hejducki, P. Rajba, M. Wodecki, Management and Production Engineering Review, 2 (2011)

2. W. Bożejko, Z. Hejducki, M. Uchroński, M. Wodecki, Journal of Civil Engineering and Management, 20(5) (2014) 